\title{
LV. On the molecular vortex theory of electromagnetic action
}

\section{R.T. Glazebrook M.A.}

To cite this article: R.T. Glazebrook M.A. (1881) LV. On the molecular vortex theory of electromagnetic action, Philosophical Magazine Series 5, 11:70, 397-413, DOI: 10.1080/14786448108627037

To link to this article: http://dx.doi.org/10.1080/14786448108627037

册 Published online: 28 Apr 2009.

Submit your article to this journal $\sqsubset \pi$

Џ Article views: 3

Q View related articles $\asymp$ 
LONDON, EDINBURGH, AND DUBLIN

\title{
PHILOSOPHICAL MAGAZINE
}

\author{
AND \\ JOURNAL OF SCIENCE. \\ [FIF'TH SERIES.] \\ JUNE 1881.
}

LV. On the Molecular Vortex Theory of Electromagnetic Action. By R. T. GlazebrooK, M.A., Fellow of Trinity College, and Demonstrator of Experimental Physics at the Cavendish Laboratory, Cambridge*.

TN Crelle's Journal, vol. lxxii., Helmholtz ("Ueber die 1 Bewegungsgleichungen der Electricität") called attention to the analogy between the equations of the electromagnetic field in a conductor and those which give the motion of a viscous fluid. The first part of the present paper is an attempt to develop more completely some of the consequences of that analogy.

Let us consider a medium in which $\xi, \eta, \zeta$ are the displacements at the time $t$, of the particle whose initial coordinates are $x, y, z$. Let $\rho$ be the density of the medium, and $\omega_{1}, \omega_{2}, \omega_{3}$ the component angular velocities of the particle about the axes, and let $\dot{\xi}=\frac{d \xi}{d t}$, \&c. Then we have

$$
\left.\begin{array}{l}
\omega_{1}=\frac{1}{2}\left\{\frac{d \dot{\xi}}{d y}-\frac{d \dot{\eta}}{d z}\right\} \\
\omega_{2}=\frac{1}{2}\left\{\frac{d \dot{\xi}}{d z}-\frac{d \dot{\zeta}}{d x}\right\}, \\
\omega_{3}=\frac{1}{2}\left\{\frac{\dot{d \eta}}{d x}-\frac{d \dot{\xi}}{d y}\right\}
\end{array}\right\}
$$

Phil. Mag. S. 5. Vol. 11. No. 70. June 1881. $2 \mathrm{G}$ 
Let

$$
\nabla^{2}=-\left\{\frac{d^{2}}{d x^{2}}+\frac{d^{2}}{d y^{2}}+\frac{d^{2}}{d z^{2}}\right\}
$$

$$
\left.\begin{array}{l}
\frac{d \omega_{3}}{d y}-\frac{d \omega_{2}}{d z}=\frac{1}{2}\left\{\nabla^{2} \dot{\xi}+\frac{d}{d x}\left(\frac{d \dot{\xi}}{d x}+\frac{d \dot{\eta}}{d y}+\frac{d \dot{\zeta}}{d z}\right)\right\} \\
\frac{d \omega_{1}}{d z}-\frac{d \omega_{3}}{d y}=\frac{1}{2}\left\{\nabla^{2} \dot{\eta}+\frac{d}{d y}\left(\frac{d \dot{\xi}}{d x}+\frac{d \dot{\eta}}{d y}+\frac{d \dot{\xi}}{d z}\right)\right\} \\
\frac{d \omega_{2}}{d z}-\frac{d \omega_{1}}{d y}=\frac{1}{2}\left\{\nabla^{2} \dot{\zeta}+\frac{d}{d z}\left(\frac{d \dot{\xi}}{d x}+\frac{d \dot{\eta}}{d y}+\frac{d \dot{\zeta}}{d z}\right)\right\}
\end{array}\right\} .
$$

Now, according to the molecular vortex theory of magnetism, magnetic force is due to vortex motion in a fluid filling space, and the magnetic force at any point is equal to the molecular rotation of the vortex at that point. Let $\alpha, \beta, \gamma$ be the magnetic force at any point. Let $\mathrm{F}, \mathrm{G}, \mathrm{H}$ be the components of the vector potential or of the electrokinetic momentum; $\dot{f, g}, \dot{h}$ the components of the electric current. Then we are to have on this hypothesis,

$$
\alpha=\omega_{1}, \quad \beta=\omega_{2}, \quad \gamma=\omega_{3} .
$$

But we know that, if $\mu$ is specific magnetic inductive capacity,

$$
\left.\begin{array}{l}
\mu \alpha=\frac{d \mathrm{H}}{d y}-\frac{d \mathrm{G}}{d z}=\mu \omega_{1}, \\
\mu \beta=\frac{d \mathrm{~F}}{d z}-\frac{d \mathrm{H}}{d x}=\mu \omega_{2}, \\
\mu \gamma=\frac{d \mathrm{G}}{d x}-\frac{d \mathrm{~F}}{d y}=\mu \omega_{3},
\end{array}\right\}
$$

Comparing these with (1), we have

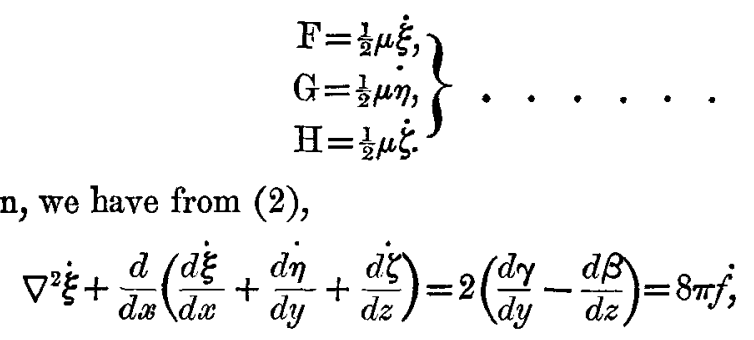

Again, we have from (2),

since (Maxwell, 'Electricity and Magnetism,’ p. 607, E)

$$
\frac{d \gamma}{d y}-\frac{d \beta}{d z}=4 \pi \dot{f}
$$


Substitute for $\dot{\xi}, \dot{\eta}, \dot{\zeta}$ the values from (4), we get

$$
4 \pi \mu \dot{f}=\nabla^{2} \mathrm{~F}+\frac{d}{d x}\left(\frac{d \mathrm{~F}}{d x}+\frac{d \mathrm{G}}{d y}+\frac{d \mathrm{H}}{d z}\right), . .
$$

and this agrees with Maxwell, p. $616(4)$.

According to the molecular vortex theory (Phil. Mag. March $1861), \mu$ is proportional to the density of the matter composing the vortices*, so that the momentum of an element parallel to the axis of $x$ will be proportional to $\mu \dot{\xi}$ or $\mathrm{F}$. Thus the momentum at any point in the direction $\mathrm{S}$ will be proportional to

$$
\mathrm{F} \frac{d x}{d s}+\mathrm{G} \frac{d y}{d s}+\mathrm{H} \frac{d z}{d s} ;
$$

and this is what Maxwell calls the electrokinetic momentum.

Let us suppose our medium to be a viscous fluid, and let $k$ be the coefficient of viscosity, $\rho$ the density of the medium, $\mathrm{X}, \mathrm{Y}, \mathrm{Z}$ the forces per unit mass, $p$ the pressure. Then we have

$$
\rho \frac{d \dot{\xi}}{d t}=\rho \mathrm{X}-\frac{d p}{d x}+\frac{1}{3} k \frac{d}{d x}\left(\frac{d \dot{\xi}}{d x}+\frac{d \dot{\eta}}{d y}+\frac{d \dot{\xi}}{d z}\right)-k \nabla^{2} \dot{\xi} .
$$

Substituting for $\dot{\xi}$ \&c. and treating $\mu$ as constant,

$$
\frac{2 \rho}{\mu} \frac{d \mathrm{~F}}{d t}=\rho \mathrm{X}-\frac{d p}{d x}+\frac{2}{3} \frac{k}{\mu} \frac{d}{d x}\left(\frac{d \mathrm{~F}}{d x}+\frac{d \mathrm{G}}{d y}+\frac{d \mathrm{H}}{d 2}\right)-\frac{2 k}{\mu} \nabla^{2} \mathrm{~F} \text {. }
$$

Substitute for $\nabla^{2} \mathrm{~F}$ from (5),

$$
\begin{aligned}
\frac{d \mathrm{~F}}{d t}=\frac{\mu}{2} & \left(\mathrm{X}-\frac{1}{\rho} \frac{d p}{d x}\right) \\
& \quad-\frac{4 \pi \mu k}{\rho} \dot{f}+\frac{4}{3} \frac{k}{\rho} \frac{d}{d x}\left(\frac{d \mathrm{~F}}{d x}+\frac{d \mathrm{G}}{d y}+\frac{d \mathrm{H}}{d z}\right) .
\end{aligned}
$$

Now let us suppose that

$$
\frac{\mu \mathrm{X}}{2}=-\frac{d \mathrm{~V}}{d x},
$$

and put

then

$$
\mathrm{V}+\frac{\mu p}{2 \rho}-\frac{4}{3} \frac{k}{\rho}\left(\frac{d \mathrm{~F}}{d x}+\frac{d \mathrm{G}}{d y}+\frac{d \mathrm{H}}{d z}\right)=\psi ; . . .
$$

$$
\frac{d \mathrm{~F}}{d t}=-\frac{d \psi}{d x}-\frac{4 \pi \mu k}{\rho} \dot{f}
$$

* If the vortices be circular and $\rho$ the density, Maxwell shows that $\rho=\frac{\mu}{2}$, so that the electrokinetic momentum is equal to

$$
\mathrm{F} \frac{d x}{d s}+\mathrm{G} \frac{d y}{d s}+\mathrm{H}_{\overline{d s}}^{d z}
$$

2 G 2 
Let $\mathrm{P}$ be the electromotive force at the point parallel to axis of $x$, and let us call $\frac{4 \pi \mu k}{\rho}$ the resistance bf the medium; then Ohm's law gives us

and we get

$$
\mathrm{P}=\frac{4 \pi \mu k}{\rho} \dot{f}
$$

Similarly

$$
\left.\begin{array}{l}
\mathrm{P}=-\frac{d \mathrm{~F}}{d t}-\frac{d \psi}{d x} . \\
\mathrm{Q}=-\frac{d \mathrm{G}}{d t}-\frac{d \psi}{d y}, \\
\mathrm{R}=-\frac{d \mathrm{H}}{d t}-\frac{d \psi}{d z} ;
\end{array}\right\}
$$

and these agree exactly with Maxwell $(598, \mathrm{~B})$, taking the case when the conductor is at rest. if

Again, substituting in (6) for $\dot{f}$ its value from (5), we get,

$$
\begin{gathered}
\mathrm{J}=\frac{d \mathrm{~F}}{d x}+\frac{d \mathrm{G}}{d y}+\frac{d \mathrm{H}}{d z}, \\
\frac{d \mathrm{~F}}{d t}+\frac{d \mathrm{~V}}{d x}+\frac{\mu}{2 \rho} \frac{d p}{d x}+\frac{k}{\rho} \nabla^{2} \mathrm{~F}-\frac{1}{3} \frac{k}{\rho} \frac{d \mathrm{~J}}{d x}=0 .
\end{gathered}
$$

Substituting and reducing,$$
\frac{d \mathrm{~F}}{d t}+\frac{d \psi}{d x}+\frac{1}{4 \pi \mu \mathrm{C}}\left(\nabla^{2} \mathrm{~F}+\frac{d \mathrm{~J}}{d x}\right)=0, \quad .
$$

C being the conductivity of the medium. And these, again, agree with Maxwell (783), supposing the medium we consider is a conductor, so that the quantity $\mathrm{K}$ in his expressions is equal to zero.

By differentiating we have

$$
\frac{d \mathrm{~J}}{d t}-\nabla^{2} \psi=0
$$

From $(8)-\frac{d \psi}{d x}$ is the electromotive force at the point parallel to $x$, so far as it does not depend on electromagnetic action, arising, that is, from the action of the free electricity in the medium. And since we consider a conductor, we have

$$
\begin{aligned}
& \nabla^{2} \psi=0 ; \\
& \therefore \frac{d J}{d t}=0 .
\end{aligned}
$$


$\mathrm{J}=\mathrm{a}$ constant $=c$ say. But the equation of continuity gives us

$$
\begin{gathered}
\mathrm{J}=-\frac{\mu}{2 \rho} \frac{d \rho}{d t}, \\
\rho=\rho_{0} e^{-\frac{2 c t}{\mu}} .
\end{gathered}
$$

And this is impossible unless $c=0$, and $\rho=$ constant; in this case $J=0$. Thus we must suppose our fluid to be incompressible.

In forming equation (7), we have put

$$
\frac{\mu \mathrm{X}}{2}=-\frac{d V}{d x}
$$

thus assuming that our forces at all points of the medium had a potential. If this be not so, let

$$
\frac{\mu \mathrm{X}}{2}=-\mathrm{P}_{1}-\frac{d \mathrm{~V}}{d x} \text {. }
$$

Then the electromotive force $\mathrm{P}_{1}$ acting at each point produces a current $f_{1}$, given by the equation

$$
\mathrm{P}_{1}=\frac{4 \pi \mu k}{\rho} \dot{f_{1}},
$$

if $\dot{f}$ is the current arising from electromagnetic action,

and

$$
f=f_{1}+f^{\prime} \text {, }
$$

$$
\mathrm{P}=\mathrm{P}_{1}+\mathrm{P}^{\prime}
$$

where $\mathrm{P}^{\prime}$ is the electromotive force arising from electromagnetic action. Thus

Again, since $\mathrm{J}=0$,

$$
\mathbf{P}^{\prime}=-\frac{d \mathrm{~F}}{d t}-\frac{d \psi}{d x}
$$

\&c.

$$
\psi=\nabla+\frac{\mu p}{2 \rho}
$$

$\psi$ is the potential of the free electricity in the medium; if the fluid be in equilibrium, the equations give us

Also

$$
\frac{d \psi}{d x}=0, \quad \frac{d \psi}{d y}=0, \quad \frac{d \psi}{d z}=0
$$

$$
\mathrm{F}=\mathrm{G}=\mathrm{H}=0 \text {. }
$$

Thus, within a conductor in which the fluid is at rest there is no electromotive force at any point. Thus the electrical phenomena that occur in a conducting medium subject to elec- 
tromagnetic action may be explained by the hypothesis that the conducting medium is filled with a viscous fluid of constant density ; forces X, Y, Z being applied to this medium at any point, rotational motion is set up.

The electromotive force parallel to the axis of $x$ at any point, so far as it depends on external electrification, is

$$
\frac{\mu}{2}\left(\mathrm{X}-\frac{1}{\rho} \frac{d p}{d x}\right)^{*}
$$

$p$ being the pressure in the fluid, and $\mu$ the magnetic specific inductive capacity. The magnetic force in the same direction is $\omega_{1}$, the angular velocity of the fluid about the axis of $x$. The electromotive force arising from electromagnetic action is

$$
-\frac{\mu}{2} \frac{d^{2} \xi^{*}}{d t^{2}}
$$

$\xi$ being the displacement of a particle of the fluid. By the action of these electromotive forces currents may be produced at any point, and the current at a point parallel to $0 x$ is

$$
\frac{1}{8 \pi} \nabla^{2}\left(\frac{d \xi}{d t}\right)
$$

If $k$ is the viscosity of the medium and $C$ its electrical conductivity, then

$$
\frac{1}{\mathrm{C}}=\frac{4 \pi \mu k}{\rho} *
$$

Now let $\mathrm{C}$ become very small; then $k$ becomes very large, the medium ceases to be a viscous fluid and becomes an elastic solid. Equations (1), (2), (3), (4), (5) hold; but instead of (6) we have

$$
\rho \frac{d \dot{\xi}}{d t}=\rho \mathrm{X}+\mathrm{A} \frac{d}{d x}\left(\frac{d \xi}{d x}+\frac{d \eta}{d y}+\frac{d \zeta}{d z}\right)-\mathrm{B} \nabla^{2} \xi . .
$$

But, integrating $(4 \mathrm{~A})$,

$$
\nabla^{2} \xi+\frac{d}{d x}\left(\frac{d \xi}{d x}+\frac{d \eta}{d y}+\frac{d \zeta}{d z}\right)=8 \pi f . . . . . .
$$

Assuming that $f$ is zero everywhere initially,

$$
\therefore \frac{d \mathrm{~F}}{d t}=\frac{\mu}{2}\left\{\mathrm{X}+\frac{\mathrm{A}+\mathrm{B}}{\rho} \frac{d}{d x}\left(\frac{d \xi}{d x}+\frac{d \eta}{d y}+\frac{d \zeta}{d z}\right)\right\}-\frac{4 \pi \mu}{\rho} \mathrm{B} f \text {. }
$$

Let

$$
\frac{\mu}{2}\left\{\mathrm{X}+\frac{\mathrm{A}+\mathrm{B}}{\rho} \frac{d}{d x}\left(\frac{d \xi}{d x}+\frac{d \eta}{d y}+\frac{d \zeta}{d z}\right)\right\}=-\frac{d \psi}{d x}
$$

* All these expressions are of oourse simplified if, with Maxwell, we put $\frac{\mu}{2}=\rho$. 
Vortex Theory of Electromagnetic Action.

let $\mathbf{P}$ be the electromotive force at the point, and suppose that

$$
\frac{\rho}{\mu \mathrm{B}}=\mathrm{K},
$$

the specific inductive capacity; then

Similarly

$$
\left.\begin{array}{rl}
\mathrm{P} & =\frac{4 \pi}{\mathrm{K}} f=\frac{4 \pi \mu \mathrm{B}}{\rho} f ; \\
\therefore \mathrm{P} & =-\frac{d \mathrm{~F}}{d t}-\frac{d \psi}{d x} \cdot \\
\mathrm{Q} & =-\frac{d \mathrm{G}}{d t}-\frac{d \psi}{d y}, \\
\mathrm{R} & =-\frac{d \mathrm{H}}{d t}-\frac{d \psi}{d z}
\end{array}\right\} \cdot . \cdot \cdot
$$

And thus in a dielectric medium also Maxwell's equations would hold.

Differentiating (12) and the two similar equations with reference to $x, y, z$ and adding, we have

$$
\frac{d f}{d x}+\frac{d g}{d y}+\frac{d h}{d z}=0 . \text {. . . . . }
$$

Differentiating (14) with reference to $t$, and substituting for $P$ from (12), remembering that

$$
\frac{\mu \dot{\xi}}{2}=\mathrm{F} \& \mathrm{c} \text {. }
$$

we have, of course, Maxwell's equations for $\mathrm{F}, \mathrm{G}, \mathrm{H}$ in a dielectric medium, viz.

$$
\mu \mathrm{K} \frac{d}{d t}\left(\frac{d \mathrm{~F}}{d t}+\frac{d \psi}{d x}\right)+\nabla^{2} \Psi+\frac{d \mathrm{~J}}{d x}=0, \& c . \quad .
$$

Maxwell, $\S 783(7)$. If $\mathrm{J}$ is a linear function of $t$, or a constant, or zero,

and

$$
\frac{d}{d x}\left(\frac{d \xi}{d x}+\frac{d \eta}{d y}+\frac{d \zeta}{d z}\right)=0
$$

$$
-\frac{d \psi}{d x}=\frac{\mu \mathrm{X}}{2}
$$

Thus the electromotive force at any point due to the free electricity is proportional to the mechanical force exerted at that point in the medium. In addition to this we have the 
electromotive force $-\frac{d \mathrm{~F}}{d t}$, which arises from the electromagnetic action. Thus, in a dielectric, electromagnetic phenomena may be explained by the strains in an elastic solid. If $K$ be the specific inductive capacity of the dielectric medium, and $B$ the coefficient of rigidity of the elastic solid,

$$
\frac{1}{\mathrm{~K}}=\frac{\mu \mathrm{B}}{\rho} .
$$

If we suppose the motion such that

$$
\frac{d \xi}{d x}+\frac{d \eta}{d y}+\frac{d \zeta}{d t}=0
$$

so that the solid is incompressible, the electric displacement at any point in the direction of $x$ is given by $\frac{1}{8 \pi} \nabla^{2} \xi$. The magnetic force is equal to the molecular rotation at that point in the solid.

In the paper in the Philosophical Magazine already referred to, Prof. Maxwell has shown that the state of stress which exists in the magnetic field is just that which would be produced by vortices in an incompressible fluid. To account for electricity, he supposes that there are a number of moving particles between these vortices. If we suppose that in a conductor Maxwell's moving medium behaves like a viscous fluid, while in a dielectric its properties are those of an elastic solid, the electrical current at any point is the "concentration" of the velocity at that point, and the electrical displacement the "concentration" of the displacement; and the additional "idle whoels" become unnecessary, except as explaining how the vortices may be conceived to rotate.

So far we have been considering the analogy between the motion of the elastic solid and electromagnetic action. Let us make the assumption that magnetic force in a dielectric arises from molecular vortices in a medium which may be treated like an elastic solid, and let us suppose that, owing to a wave of displacement travelling through this medium, the vortices are displaced, and a term arises in the kinetic energy of the form

$$
2 \overline{\mathrm{C}}\left(\alpha \omega_{1}+\beta \omega_{2}+\gamma \omega_{3}\right),
$$

$\omega_{1}, \omega_{2}, \omega_{3}$ being the angular velocities of the element considered which arise from the displacement $\xi, \eta, \zeta$. Then Maxwell has shown that the kinetic energy $\mathrm{T}$ per unit volume is given by 
where

$$
\begin{aligned}
\mathrm{T}=\frac{1}{2} \rho\left(\dot{\xi}^{2}\right. & \left.+\dot{\eta^{2}}+\dot{\zeta^{2}}\right)+\overline{\mathrm{C}}\left\{\dot{\xi} \frac{d}{d \theta}\left(\frac{d \xi}{d y}-\frac{d \eta}{d z}\right)\right. \\
& \left.+\dot{\eta} \frac{d}{d \theta}\left(\frac{d \xi}{d z}-\frac{d \zeta}{d x}\right)+\dot{\zeta} \frac{d}{d \theta}\left(\frac{d \eta}{d x}-\frac{d \xi}{d y}\right)\right\}, .
\end{aligned}
$$

$$
\frac{d}{d \theta}=\alpha \frac{d}{d x}+\beta \frac{d}{d y}+\gamma \frac{d}{d z} ;
$$

and from this he has obtained an expression for the rotation of the plane of polarization of light in the field.

I wish to put this in a somewhat more general form, in order to develop the connexion between it and the electromotive force discovered experimentally by Mr. E. H. Hall (Phil. Mag. March 1880), which leads, as Prof. Rowland has shown, to the same expression for the rotation of the plane of polarization of the light. Let us suppose, then, that forces $\mathrm{X}, \mathrm{Y}, \mathrm{Z}$ are applied per unit mass at each point of our medium, and also that we may consider it as incompressible. Then, if $\mathrm{W}$ be the potential energy and $B$ the coefficient of rigidity,

$-\mathrm{W}=\rho(\mathrm{X} \delta \xi+\mathrm{Y} \delta \eta+\mathrm{Z} \delta \xi)$

$$
\begin{aligned}
+ & \frac{\mathrm{B}}{2}\left\{\left(\frac{d \xi}{d y}+\frac{d \eta}{d x}\right)^{2}+\left(\frac{d \xi}{d z}+\frac{d \xi}{d x}\right)^{2}+\left(\frac{d \eta}{d z}+\frac{d \zeta}{d y}\right)^{2}\right. \\
& \left.-4\left(\frac{d \eta}{d y} \frac{d \zeta}{d z}+\frac{d \xi}{d x} \frac{d \zeta}{d z}+\frac{d \xi}{d x} \frac{d \eta}{d y}\right)\right\} . . .
\end{aligned}
$$

To obtain the equations of motion we follow the method adopted by Fitzgerald (" On the Electromagnetic Theory of the Reflexion and Refraction of Light," Phil. Trans. 1880); and from the condition that

we arrive finally at the equations

$$
\delta \int(\mathrm{T}+\mathrm{W}) d t=0,
$$

$$
\rho \ddot{\xi}+2 \overline{\mathrm{C}} \frac{d}{d \theta}\left(\frac{d \dot{\xi}}{d y}-\frac{\dot{d} \boldsymbol{\eta}}{d z}\right)=\rho \mathrm{X}-\mathrm{B} \nabla^{2 \xi} \& \mathrm{c} .
$$

Now we have seen already that, on the molecular vortex theory, if $\alpha^{\prime}, \beta^{\prime}, \gamma^{\prime}$ denote the magnetic force, $f, g, h$ the electric displacement, and $\mathrm{F}, \mathrm{G}, \mathrm{H}$ the components of vector potential due to the displacements considered,

$$
\begin{aligned}
& \alpha^{\prime}=\omega_{1}=\frac{1}{2}\left(\frac{d \dot{\zeta}}{d y}-\frac{d \dot{\eta}}{d z}\right) \& c . \\
& f=\frac{1}{8 \pi} \cdot \nabla^{2} \xi \& \mathrm{c} . \\
& \mathrm{F}=\frac{\mu \dot{\xi}}{2} \& \mathrm{c} .
\end{aligned}
$$


Thus, substituting for $\xi \& c$.,

Now

$$
\begin{aligned}
& \frac{d \mathrm{~F}}{d t}+\frac{2 \mu \overline{\mathrm{C}}}{\rho} \frac{d \alpha^{\prime}}{d \theta}=\frac{\mu \mathrm{X}}{2}-\frac{4 \pi \mathrm{B} \mu}{\rho} f . \\
& \frac{d \alpha^{\prime}}{d \theta}=\alpha \frac{d \alpha^{\prime}}{d x}+\beta \frac{d \alpha^{\prime}}{d y}+\gamma \frac{d \alpha^{\prime}}{d z} \\
= & \alpha \frac{d \alpha^{\prime}}{d x}+\beta \frac{d \beta^{\prime}}{d x}+\gamma \frac{d \gamma^{\prime}}{d x}+4 \pi(\gamma \dot{g}-\beta \dot{h}) \\
= & \frac{d}{d x}\left(\alpha \alpha^{\prime}+\beta \beta^{\prime}+\gamma \gamma^{\prime}\right)+4 \pi(\gamma \dot{g}-\beta \dot{h}),
\end{aligned}
$$

since $\alpha, \beta, \gamma$ are constant. Put $-\frac{d \psi}{d x}$ for $\frac{\mu \mathrm{X}}{2}$, the total electromotive force impressed on the element parallel to the axis of $x$, and, as before, lot

$$
\frac{\mu \mathrm{B}}{\rho}=\frac{1}{\mathrm{~K}}
$$

$K$ being the specific inductive capacity; then, if $P$ be the electromotive force parallel to $x$,

Then

$$
\mathrm{P}=\frac{4 \pi f}{\mathrm{~K}} \text {. }
$$

$$
\begin{aligned}
\frac{d \mathrm{~F}}{d t}+\frac{8 \pi \mu \overline{\mathrm{C}}}{\rho}(\gamma \dot{g}-\beta \dot{h})+\frac{2 \mu \overline{\mathrm{C}}}{\rho} \frac{d}{d x}\left(\alpha \alpha^{\prime}\right. & \left.+\beta \beta^{\prime}+\gamma \gamma^{\prime}\right) \\
& +\mathrm{P}=-\frac{d \psi}{d x} .
\end{aligned}
$$

Thus $\mathrm{P}$, the electromotive force parallel to $x$, is, on the molecular vortex theory, composed of four parts in a field of strong magnetic force. The first is $-\frac{d \mathrm{~F}}{d t}$, the rate of change of the vector potential. The next is

$$
-\frac{\delta \pi \mu \overline{\mathrm{C}}}{\rho}(\gamma \dot{g}-\beta \dot{h})
$$

and this is exactly similar in form to the electromotive force discovered by Mr. E. H. Hall and already alluded to. The third may be written

$$
-\frac{2 \mu \overline{\mathrm{C}}}{\rho} \frac{d}{d x}\left(\mathfrak{h} \mathfrak{h}^{\prime} \cos \epsilon\right) ;
$$

where $\mathfrak{b}$ is the impressed magnetic force, $\mathfrak{y}^{\prime}$ the resultant of the force arising from the displacement of the medium, and $\epsilon$ the angle between them. In Mr. Hall's experiments this angle would be $90^{\circ}$, and therefore the term would vanish. 
The fourth term $-\frac{d \psi}{d x}$ is, of course, that part of the electromotive force which arises from the electricity in the field. Had we considered the case of a conductor, the terms which we should have to add to equations (8) would be just the same as those added above in (20) to equation (14). Thus, in a conductor we should have

$$
\begin{gathered}
\frac{d \mathrm{E}}{d t}+\frac{8 \pi \mu \overline{\mathrm{C}}}{\rho}(\gamma \dot{g}-\beta \dot{h})+\frac{2 \mu \overline{\mathrm{C}}}{\rho} \frac{d}{d x}\left(\alpha \alpha^{\prime}+\beta \beta^{\prime}+\gamma \gamma^{\prime}\right) \\
\& \mathrm{c} . \quad+\mathrm{P}=-\frac{d \psi}{d x} .
\end{gathered}
$$

In the case considered by Mr. Hall,

and

$$
\frac{d \mathrm{~F}}{d t}=0, \quad \alpha \alpha^{\prime}+\beta \beta^{\prime}+\gamma \gamma^{\prime}=0,
$$

$-\frac{d \psi}{d x}=$ impressed electromotive force in direction of $x=\mathrm{P}_{1}$ say;

$$
\therefore \mathrm{P}=\mathrm{P}_{1}-\frac{8 \pi \mu \overline{\mathrm{C}}}{\rho}(\dot{\gamma g}-\beta \dot{h}) . \quad . \quad .
$$

Thus, if a current $\dot{f}$ flow in a conductor in a field of strong magnetic force $\gamma$, there will be produced an electromotive force in the direction of $y$ (perpendicular, that is, to the directions of $j$ and $\gamma$ ), whose value will bo

$$
+\frac{8 \pi \mu \overline{\mathrm{C}}}{\rho} \gamma \dot{f} \text {. }
$$

If we consider a wave travelling through the medium, the electromotive force parallel to $x$ produced by electromagnetic action will be

$$
-\frac{d \mathrm{~F}}{d t}-\frac{8 \pi \mu \overline{\mathrm{C}}}{\rho}(\gamma \dot{g}-\beta \dot{\mathrm{h}}) .
$$

Substituting in the equations

we get

$$
-\mu \mathrm{K} \frac{d \mathrm{P}}{d t}+\nabla^{2} \mathrm{~F}=0
$$

$$
\mu \mathrm{K}\left\{\frac{d^{2} \mathrm{~F}}{d t^{2}}+\frac{8 \pi \mu \widetilde{\mathrm{C}}}{\rho} \frac{d}{d t}(\gamma \dot{g}-\beta \dot{h})\right\}+\nabla^{2} \mathrm{~F}=0 ;
$$

and from these Professor Rowland has calculated the magnetic rotation of the vectors F, G, H (Phil. Mag. April 1881).

The equations satisfied by the magnetic force $\alpha^{\prime}, \beta^{\prime}, \gamma^{\prime}$, can be found either from these by differentiation, or from the original equations (19). We get, remembering that

$$
\dot{f}=\frac{1}{4 \pi}\left(\frac{d \gamma^{\prime}}{d y}-\frac{d \beta^{\prime}}{d z}\right)
$$




$$
\begin{gathered}
\mu \mathrm{K}\left\{\frac{d^{2} \alpha^{\prime}}{d t^{2}}+\frac{2 \overline{\mathrm{C}}}{\rho} \frac{d}{d t}\left(\alpha \frac{d}{d x}+\beta \frac{d}{d y}+\gamma \frac{d}{d z}\right)\left(\frac{d \gamma^{\prime}}{d y}-\frac{d \beta^{\prime}}{d z}\right)\right\} \\
+\nabla^{2} \alpha^{\prime}=0,
\end{gathered}
$$

These or the original equations (19), if we put $\frac{\mathrm{B}}{\rho}=\frac{1}{\mu \mathrm{K}}$, and $\mathrm{X}$ the impressed electromotive force $=0$, and substitute for $\frac{2 \mu \overline{\mathrm{C}}}{\rho}, 8 \pi \mathrm{C}$, agree with $\mathrm{Mr}$. Fitzgerald's equation. If we consider that the magnetic force is parallel to $z$ and that our wave is travelling in that direction, Mr. Fitzgerald has shown that the wave must consist of two circularly polarized waves travelling respectively with velocities $v_{1}$ and $v_{2}$, where

and

$$
v_{1}=\frac{1}{\sqrt{\mu \mathrm{K}}}+\frac{2 \pi \overline{\mathrm{C}} \gamma}{\rho \bar{\lambda}},
$$

$$
v_{2}=\frac{1}{\sqrt{\mu \mathrm{K}}}-\frac{2 \pi \overline{\mathrm{C}} \gamma}{\rho \lambda}
$$

So that the rotation of the plane of polarization produced by a length $l$ of the substance is

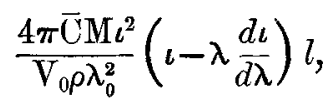

$\iota$ being the refractive index, $V_{0}$ and $\lambda_{0}$ the velocity and wavelength in air, and $M$ the magnetic force. The rotation of unit length for unit magnetic force will be $\frac{4 \pi \mathrm{C} \iota^{3}}{\mathrm{~V}_{0} \rho \lambda_{0}^{2}}$ if we neglect $\frac{d \iota}{d \lambda}$. According to Mr. J. E. H. Gordon (Phil. Trans. 1877), the value of this quantity for the thallium-ray in carbon disulphide is $3.047 \times 10^{-5}$ about. Also

$$
\begin{aligned}
\lambda_{0} & =5.349 \times 10^{-5}, \\
V_{0} & =3 \times 10^{10}, \\
V_{0} \lambda_{0}^{2} & =75 ;
\end{aligned}
$$

$\rho$ is the density of the medium-of course not the dielectric, but the æther. According to Maxwell ("Physical Lines of Force," Phil. Mag. April 1861), this is of the same order as $\mu$, being equal to $\frac{\mu}{2}$ if the vortices are circular, and $\mu$ is nearly unity. Thus

$$
\overline{\mathrm{C}}=\frac{3.047 \times 10^{-5} \times 75}{4 \pi \iota^{3}} ;
$$

and $\bar{C}$ is of order

$$
10^{-5} \text { or } 10^{-6} \text {. }
$$


In his paper Prof. Rowland remarks, after showing that Mr. Hall's discovery leads to the same expressions for the rotation of the plane as Prof. Maxwell's, "the conclusion we draw is that the effect discovered by Mr. Hall is the same or due to the same cause as the rotation of the plane of polarization of light." The previous investigation, 1 think, may supply a connecting link between the two.

As has already been stated, equations (19) and (23) agree in form with Mr. Fitzgerald's equations ; and in fact (23) is identical with his. For let us put

$$
\dot{p}=\alpha^{\prime}, \quad \dot{q}=\beta^{\prime}, \quad \dot{r}=\gamma^{\prime} ;
$$

then $p, q, r$ are the same as Mr. Fitzgerald's $\xi, \eta, \zeta$, and his final equation is

$$
\begin{gathered}
\mu k\left\{\frac{d^{2} p}{d t^{2}}+8 \pi \mathrm{C} \frac{d}{d t}\left(\alpha \frac{d}{d x}+\beta \frac{d}{d y}+\gamma \frac{d}{d z}\right)\left(\frac{d r}{d y}-\frac{d q}{d r}\right)\right\} \\
\text { de. } ; \quad+\nabla^{2} p=0, . .
\end{gathered}
$$

and differentiating these with respect to $t$ and substituting, we get (23).

Now Mr. Fitzgerald's equation has been deduced, without any assumption as to the connexion between the motions of matter and wther, from the hypothesis that the electrokinetic energy of the medium contains a term

where

$$
4 \pi \mathrm{C} \iiint\left\{\frac{d p}{d \bar{\theta}} \dot{f}+\frac{d q}{d \theta} \dot{g}+\frac{d r}{d \theta} \dot{h}\right\} d x d y d z,
$$

$$
\frac{d}{d \theta}=\alpha \frac{d}{d x}+\beta \frac{d}{d y}+\gamma \frac{d}{d z}
$$

Professor Maxwell's additional term depends on the displacements of the medium which constitute light. The potential energy in his case is that which arises from the state of strain of the medium, while in Mr. Fitzgerald's paper the potential energy is the electrostatic energy of the field. The foregoing investigation shows that the two assumptions lead to the same equations for the electric and magnetic disturbance.

If we suppose that we have in the field light-motion in addition to electrical and magnetic displacements, and assume with Prof. Maxwell his additional term in the kinetic energy, then, on the molecular vortex theory, the electrokinetic energy, or that part of the kinetic energy on which electrical and magnetic effects depend, will contain a term of the form supposed by Mr. Fitzgerald. The kinetic energy being

$$
\frac{\rho}{2} \iiint\left(\dot{\xi}^{2}+\dot{\eta}^{2}+\dot{\zeta}^{2}\right) d x d y d z
$$


the electrokinetic is

$$
\frac{\mu}{8 \pi} \iiint\left(\dot{p}^{2}+\dot{q}^{2}+\dot{r}^{2}\right) d x d y d z
$$

being obtained from the kinetic by substituting $\frac{\mu}{4 \pi}$ for $\rho$, and $p, q, r$ for $\xi, \eta, \zeta$. If, then, in the kinetic energy we have the term

$$
\overline{\mathrm{C}}\left\{\dot{\xi} \frac{d}{d \theta}\left(\frac{d \zeta}{d y}-\frac{d \eta}{d z}\right)+\& \mathrm{c} .\right\} d x d y d z,
$$

in the electrokinetic energy we have

$$
\overline{\mathrm{C}}\left\{\dot{p} \frac{d}{d \theta}\left(\frac{d r}{d y}-\frac{d q}{d z}\right)+\& c .\right\} d x d y d z ;
$$

and this, on integrating by parts, gives

$$
4 \pi \overline{\mathrm{C}}\left\{\dot{f} \frac{d p}{d \theta}+\dot{g} \frac{d q}{d \theta}+\dot{h} \frac{d r}{d \theta}\right\} d x d y d z,
$$

which is Mr. Fitzgerald's additional term.

In fact, if we consider the medium as consisting of a number of separate molecules, the kinetic energy has its ordinary meaning; the electrokinetic is that part of the kinetic energy which depends only on the rotation of the molecules; for this rotation alone produces magnetic force. If a transverse wave of disturbance travels through the medium, the translational motion of each molecule becomes known to us as light, while to the rotational velocity we give the name of magnetic force. Since the direction of rotation at each point is reversed many times a second, we cannot produce magnetic force by a wave of light. If, however, magnetic force exists in the medium independently of the light, the translational motion of the molecules is modified thereby.

Now let us consider a molecule of the medium, which we shall suppose moves as a rigid body, with angular velocities $\omega_{1}, \omega_{2}, \omega_{3}$. If $\xi, \eta, \zeta$ are the coordinates of the centre of gravity, and $d x d y d z$ the volume, $\mathrm{A}, \mathrm{B}, \mathrm{C}$ the radii of gyration about axes parallel to those of coordinates, which we assume to be principal axes, then the kinetic energy of this molecule is

$$
\frac{1}{2} \rho\left(\dot{\xi}^{2}+\dot{\eta}^{2}+\dot{\zeta}^{2}\right) d x d y d z+\rho\left(\mathrm{A}^{2} \omega_{1}^{2}+\mathrm{B}^{2} \omega_{2}^{2}+\mathrm{C}^{2} \omega_{3}^{2}\right) d x d y d z .
$$

In a material medium, $A, B, C$ being proportional to the linear dimensions of the molecule, the last term, $A^{2} \omega_{1}^{2}+B^{2} \omega_{2}^{2}+C^{2} \omega_{3}^{2}$, vanishes compared with the other, the moleculo being very 
small. Let us suppose that in the æther, $\omega_{1}, \omega_{2}, \omega_{3}$ become known to us as magnetic force; then this last term expresses the magnetic energy in the field. And if we suppose, further, that

$$
\mathrm{A}^{2}=\mathrm{B}^{2}=\mathrm{C}^{2}=\frac{\mu}{8 \pi \rho}
$$

since $\omega_{1}=\alpha \& c$, the magnetic energy of the whole field may be written

$$
\frac{\mu}{8 \pi} \iiint\left(\alpha^{2}+\beta^{2}+\gamma^{2}\right) d x d y d z ;
$$

and this may be transformed to

$$
\frac{\mu}{16 \pi} \iiint\left\{\dot{\xi} \nabla^{2} \dot{\xi}+\dot{\eta} \nabla^{2} \dot{\eta}+\dot{\zeta} \nabla^{2} \dot{\zeta}\right\} d x d y d z
$$

by an application of Green's theorem whenever $\xi, \eta, \zeta$ are functions of the same function of $x, y, z$. Hence, remembering that

$$
\nabla^{2} \dot{\zeta}=8 \pi \dot{f}, \quad \frac{\mu \dot{\zeta}}{2}=\mathrm{F}, \dot{f}=u \& c .
$$

the electrokinetic energy becomes

$$
\iiint(\mathrm{F} u+\mathrm{G} v+\mathrm{H} w) d x d y d z .
$$

Since in the kinetic energy of the field we have, on the molecular vortex theory, a term involving $\left(\omega_{1}^{2}+\omega_{2}^{2}+\omega_{3}^{2}\right)$, we see that if we put for $\omega_{1}$

$$
\alpha+\omega_{1},
$$

$\alpha$ being the rotation which constitutes the magnetic force, and $\omega_{1}$ the rotation due to the wave of displacement travelling through, we shall introduce a term $\alpha \omega_{1}+\beta \omega_{2}+\gamma \omega_{3}$, which is the hypothesis from which we started.

If we start from Mr. Fitzgerald's standpoint, and assume terms in the electrokinetic energy of the form

$$
4 \pi \mathrm{C} \iint\left(\dot{f} \frac{d p}{d \theta}+\dot{g} \frac{d q}{d \theta}+\dot{h} \frac{d r}{d \theta}\right) d x d y d z
$$

to explain the phenomena of the rotation of the plane of polarization without reference to theories of molecular vortices, we may show that this assumption also leads to Hall's effect. We wish to transform this term into one in which the kinetic energy is expressed in terms of $f, g, h, \dot{f}, \dot{g}, \dot{h}$ and constants. Then, if $\mathrm{T}$ be the kinetic energy, and $\mathrm{P}, \mathrm{Q}, \mathrm{R}$ the components 
Now

of the electromotive force,

$$
\left.\begin{array}{l}
-\mathrm{P}=\frac{d}{d t} \frac{d \mathrm{~T}}{d \dot{f}}-\frac{d \mathrm{~T}}{d f}, \\
-\mathrm{Q}=\frac{d}{d t} \frac{d \mathrm{~T}}{d \dot{g}}-\frac{d \mathrm{~T}}{d g}, \\
-\mathrm{R}=\frac{d}{d t} \frac{d \mathrm{~T}}{\dot{h}}-\frac{d \mathrm{~T}}{d h}
\end{array}\right\} \cdot . \cdot \cdot
$$

$$
\iiint \dot{f} \frac{d p}{d \theta} d x d y d z=\iiint \dot{f}\left(\alpha \frac{d p}{d x}+\beta \frac{d p}{d y}+\gamma \frac{d p}{d z}\right) d x d y d z .
$$

But we have, from the definitions of $p, q, r$,

$$
\begin{aligned}
& \frac{d p}{d y}=\frac{d g}{d x}-4 \pi h \\
& \frac{d p}{d z}=\frac{d r}{d x}+4 \pi g .
\end{aligned}
$$

Thus the term in $\dot{f}$ becomes

$$
\begin{aligned}
& 4 \pi \iiint f(g \gamma-h \beta) d x d y d z \\
& \quad+\iiint f\left(\alpha \frac{d p}{d x}+\beta \frac{d q}{d x}+\gamma \frac{d r}{d x}\right) d x d y d z .
\end{aligned}
$$

Integrate the last term by parts ; then, if we neglect the surface-integral, we have

$$
-\iiint\left[p \frac{d}{d x}(\alpha \dot{f})+q \frac{d}{d x}(\beta \dot{f})+r \frac{d}{d x}(\gamma \dot{f})\right] d x d y d z
$$

and the additional term in the kinetic energy becomes

$$
\begin{aligned}
16 \pi^{2} \mathrm{C} \iiint[\dot{f}(\gamma g-\beta h)+\dot{g}(\alpha h-\gamma f)+\dot{h}(\beta f-\alpha g)] d x d y d z \\
\quad-4 \pi \mathrm{C} \iiint\left[p\left\{\frac{d}{d x}(\alpha \dot{f})+\frac{d}{d y}(\alpha \dot{g})+\frac{d}{d s}(\alpha \dot{h})\right\}\right. \\
+q\left\{\frac{d}{d x}(\dot{\beta} \dot{f})+\frac{d}{d y}(\beta \dot{g})+\frac{d}{d z}(\beta \dot{h})\right\} \\
\left.+r\left\{\frac{d}{d x}(\gamma \dot{f})+\frac{d}{d y}(\gamma \dot{g})+\frac{d}{d z}(\gamma \dot{h})\right\}\right] d x d y d z .
\end{aligned}
$$

The coefficient of $p$ in the last, on substituting for $\dot{f}, \dot{g}, \dot{h}$ the values

$$
\frac{1}{4 \pi}\left(\frac{d \gamma}{d y}-\frac{d B}{d z}\right) \& c
$$


and reducing, becomes

$$
\frac{d \alpha}{d x} \frac{d \gamma}{d y}-\frac{d \alpha}{d y} \frac{d \gamma}{d x}+\frac{d \alpha}{d z} \frac{d \beta}{d x}-\frac{d \alpha}{d x} \frac{d \beta}{d z}
$$

And this expression vanishes whenever $\alpha, \beta, \gamma$ are functions of the same function of $x, y$, and $z$. This is, of course, satisfied if $\alpha, \beta, \gamma$ are constants or components of a wave of magnetic force crossing the medium.

Hence, whenever a wave is traversing a field of magnetic force, the electrokinetic energy is

$$
\begin{aligned}
\iiint\left[\mathrm{F} \dot{f}+\mathrm{G} \dot{g}+\mathrm{H} \dot{h}+16 \pi^{2} \mathrm{O}\{\dot{f}(\gamma g-\beta h)+\dot{g}(\alpha h-\gamma f)\right. \\
+\dot{h}(\beta f-\alpha g)\}] d x d y d z .
\end{aligned}
$$

The terms in $\mathrm{C}$ are in general small; and if, with Mr. Fitzgerald, we are considering a wave in a field of strong uniform magnetic force components $\bar{\alpha}, \bar{\beta}, \bar{\gamma}$, we may put $\bar{a}, \bar{\beta}, \bar{\gamma}$ for $\alpha, \beta, \gamma$ in the above terms and treat them as constant. Then, from the equations

we get

$$
\mathrm{P}=-\frac{d}{d t} \frac{d \mathrm{~T}}{d \dot{f}}-\frac{d \mathrm{~T}}{d f}
$$

$$
\left.\begin{array}{l}
\mathrm{P}=-\frac{d \mathrm{~F}}{d t}-32 \pi^{2} \mathrm{C}(\gamma \dot{g}-\beta \dot{h}), \\
\mathrm{Q}=-\frac{d \mathrm{G}}{d t}-32 \pi^{2} \mathrm{C}(\alpha \dot{h}-\gamma \dot{f}), \\
\mathrm{R}=-\frac{d \mathrm{H}}{d t}-32 \pi^{2} \mathrm{C}(\beta \dot{f}-\alpha \dot{g}) .
\end{array}\right\} . \cdot
$$

And these agree exactly with (21), remembering that

$$
\frac{\mu \overline{\mathrm{C}}}{\rho}=4 \pi \mathrm{C}
$$

and that in the present case we have neglected quantities like $\alpha^{\prime}, \beta^{\prime}, \gamma^{\prime}$. Thus, the additional term assumed by Mr. Fitzgerald leads to Mr. Hall's additional terms in the electromotive force. Of course, if we start from Mr. Hall's terms in the electromotive force, and work backwards to find the electrokinetic energy, we shall arrive at Mr. Fitzgerald's term; and if, further, we assume the hypotheses of the molecular vortex theory, we shall get Maxwell's additional term. $\mathrm{Mr}$. Fitzgerald's term is a direct consequence of Hall's experiments; Maxwell's term is a consequence of them on some theory of the action between light and magnetism.

Phil. Mag. S. 5. Vol. 11. No. 70. June 1881. $2 \mathrm{H}$ 